\title{
On the analysis and detection of flames with an asynchronous spiking image sensor
}

\author{
Juan A. Leñero-Bardallo, José M. Guerrero-Rodríguez, Ricardo Carmona-Galán, and Ángel Rodríguez-Vázquez
}

\begin{abstract}
We have investigated the capabilities of a custom asynchronous spiking image sensor operating in the Near Infrared (NIR) band to study flame radiation emissions, monitor their transient activity, and detect their presence. Asynchronous sensors have inherent capabilities, i.e. good temporal resolution, high dynamic range, and low data redundancy. This makes them competitive against Infrared (IR) cameras and CMOS frame-based NIR imagers. In the article, we analyze, discuss and compare the experimental data measured with our sensor against results obtained with conventional devices. A set of measurements have been taken to study the flame emission levels and their transient variations. Moreover, a flame detection algorithm, adapted to our sensor asynchronous outputs, has been developed. Results show that asynchronous spiking sensors have an excellent potential for flame analysis and monitoring.
\end{abstract}

Index terms -Flame monitoring; NIR sensors; CMOS; Asynchronous Image Sensors; AER; Luminance sensors; Octopus. ${ }^{1}$

\section{INTRODUCTION}

The detection and the analysis of flames activity are necessary in industrial and scientific environments. Flame flickering and flame emissions have to be monitored in some industrial processes [1], [2]. For obvious reasons, flame detection is crucial in many situations. These tasks have been traditionally implemented with infrared (IR) cameras or microbolometers. Such devices are effective detecting hot spots and temperature variations within the visual scene. However, they are still relatively expensive, they require calibration in some cases, and their speed response is limited, [3].

As an alternative to infrared detectors, CMOS or CCD cameras operating in the Near Infrared (NIR) band can be used in some specific application scenarios [4]-[7]. Placing a NIR optical filter over them, they become NIR imagers. Hot spots, fire or flames can be detected with a CMOS sensor with a NIR filter [5], [8]. Regions whose temperature is above $350^{\circ} \mathrm{C}$ can be identified [5]. Smoke detection is also possible employing NIR sources, e.g. NIR LEDs, [5]. However, their speed response is low for real-time flame activity monitoring [1]. Also, the intra-scene dynamic range of an average CMOS

\footnotetext{
${ }^{1}$ This work was supported in part by the University of Cádiz under Grant PR2016-072; in part by the Spanish Ministry of Economy and Competitiveness under Grant TEC2015-66878-C3-1-R, Co-Funded by ERDF-FEDER; in part by Junta de Andalucía CEICE under Grant TIC 2012-2338 (SMARTCIS3D); and in part by ONR under Grant N000141410355 (HCELLVIS).

Juan A. Leñero-Bardallo, R. Carmona-Galán, and Á. Rodríguez-Vázquez are with the Institute of Microelectronics of Seville (IMSE-CNM), CSICUniversidad de Sevilla, Spain (E-mails: \{juanle,rcarmona,angel\}@imsecnm.csic.es).

José M. Guerrero-Rodríguez are with the University of Cádiz, Spain, (E-mail: josem.guerrero@uca.es).
}

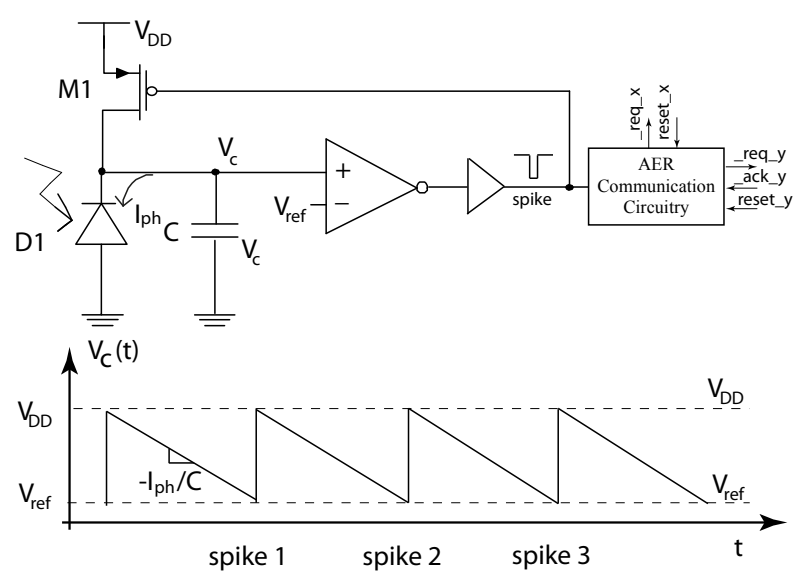

Fig. 1. Integrate-and-fire pixel block diagram. Pixels spike with a frequency proportional to light intensity. Every time that this occurs, pixels' addresses are transmitted off-chip with the AER communication protocol. Asynchronous digital signals involved in the data transmission are displayed.

sensor is usually below 70dB [9], not being suitable to gauge precisely energy variations inside very bright light sources.

Recently, asynchronous image sensors working in the NIR band were introduced by the authors, as an alternative to CMOS cameras with NIR filters [10]. Event-based spiking luminance sensors, also known as octopus retinas [11]-[13], have some inherent advantages over conventional frame-based sensors: fast operation, high dynamic range operation, and low output data throughout, i.e. only illuminated pixels send data out. Therefore, they are good candidates to perform the tasks previously described. If we place over them a NIR filter, they become asynchronous luminance sensors operating in the NIR band. They inherit two very important features to study flames: good temporal resolution and high dynamic range. Fast operation is necessary because flames flicker with frequency components up to $100 \mathrm{~Hz}$ [1]. High Dynamic Range (HDR) operation is also mandatory because flames are very bright sources; radiation emitted by a flame depends on the location inside it [3].

In this article, we analyze, assess, and compare the performance of NIR spiking sensors when monitoring flame activity or detecting flames. In the first part of the manuscript, we compare their performance studying the variation of energy levels in flames against a commercial infrared camera. In the second part, we focus on the measurement of transient variations of NIR radiation due to flame flickering. Based on their properties, we propose an algorithm to detect the presence of flames with a spiking image sensor operating in 
(a)

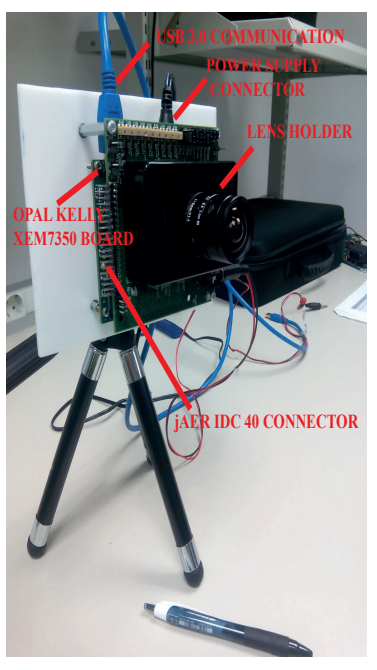

(b)

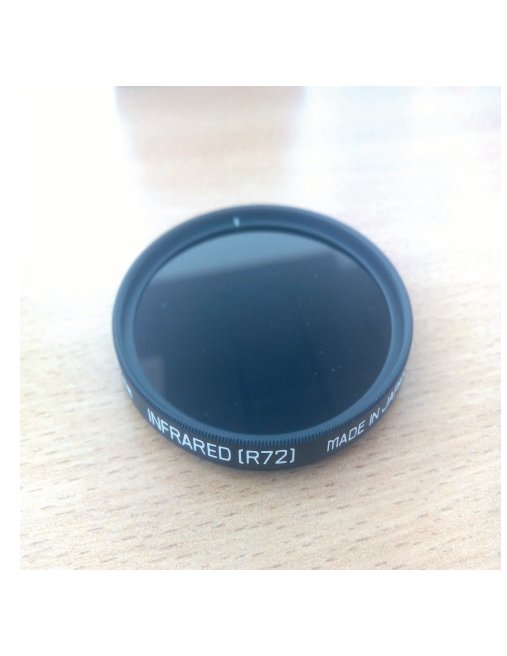

Fig. 2. Experimental setup. (a) Sensor's implementation and its optics. (b) NIR optical filter.

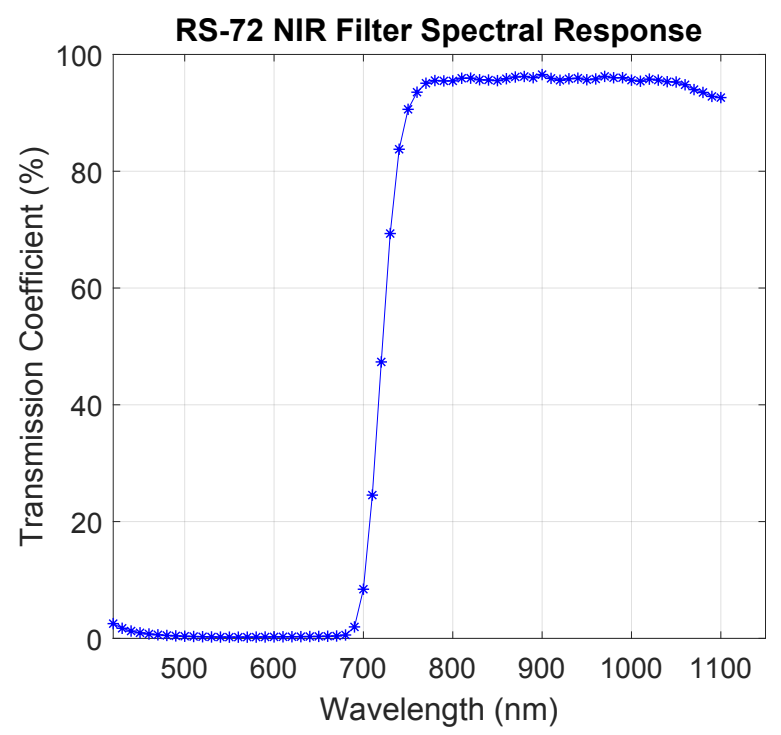

Fig. 3. Measured transfer function of the RS-72 NIR optical filter.

the NIR band.

\section{NIR SENSOR IMPLEMENTATION}

We built an asynchronous luminance sensor operating in the NIR band. We used a previously reported and designed CMOS spiking image sensor [14]. The device has several operation modes. It can be configured to operate continuously, generating spikes with a frequency proportional to illumination. In this operation mode, the pixel blocks involved in the light-tofrequency conversion are shown in Fig. 1. Initially, the voltage at the integration capacitance, $V_{C}$, is reset and its value is closer to $V_{D D}$. Then, the voltage decreases with a slope that is proportional to illumination. Whenever the voltages reaches the value $V_{r e f}$, the pixel self-resets: the $V_{C}$ voltage is set
TABLE I

SPIKING NIR SENSOR FEATURES

\begin{tabular}{|c|c|}
\hline Technology & AMS $0.18 \mu \mathrm{m}$ HV \\
\hline Power Supply & $1.8 \mathrm{~V} / 5 \mathrm{~V}$ \\
\hline Chip Dimensions & $4120 \mu \mathrm{m} \times 3315 \mu \mathrm{m}$ \\
\hline Pixel Size & $25 \mu \mathrm{m} \times 25 \mu \mathrm{m}$ \\
\hline Number of Pixels & $96 \times 128$ \\
\hline Fill Factor & $10 \%$ \\
\hline Latency & $<5 \mathrm{~ms} @ 1 \mathrm{klux}$ or lower \\
\hline Dynamic Range & $>100 \mathrm{~dB}$ \\
\hline Event sensitivity & $0.0762 \mathrm{events} / \Delta V \cdot l u x$ \\
\hline Power Consumption & $52 \mathrm{~mW} @ 100 \mathrm{keps}$ \\
\hline Sense Node Capacitance & $45 \mathrm{fF}$ \\
\hline FPN (event output) & $2.6 \%$ \\
\hline Max. event rate & $2 \mathrm{Meps}$ (same row), \\
& $10 \mathrm{Meps}$ (different rows) \\
\hline
\end{tabular}

again to $V_{D D}$ and the pixel's coordinates are transmitted offchip using the AER (Address Event Representation) protocol [15]. The reception of a pixel address off-chip is called event. Specific asynchronous circuitry [12], [16] is added in-pixel and on the sensor array periphery to implement the asynchronous communication. For the sake of simplicity, we will not detail it again in this publication.

Examining the pixel design, it can be easily demonstrated that the pixel firing frequency is giving by this equation:

$$
f_{o s c} \approx \frac{I_{p h}}{C \cdot\left(V_{D D}-V_{\text {ref }}\right)}=\frac{I_{p h}}{C \cdot \Delta V}
$$

Where $C$ is the pixel integration capacitance, $I_{p h}$ is the local photocurrent, and $\Delta V$ is the voltage difference between $V_{D D}$ and an adjustable programmable voltage threshold $V_{\text {ref }}$.

The sensor was implemented in standard CMOS technology. Hence it can sense all the radiation within the visible spectrum and NIR radiation up to $1100 \mathrm{~nm}$. To restrict the sensor operation to the NIR band, we placed over its optic an optical HP filter that rejects all the radiation below the NIR band. The experimental setup and the filter are shown in Fig. 2. There is an FPGA attached to the sensor to send data to a PC and to configure the sensor's operation with a custom interface [14]. Optionally, a data logger can also be connected through an IDC40 connector to the sensor. The filter transfer curve was characterized in our laboratories with a monochromator and an optical power meter. Results are displayed in Fig. 3. Radiation below $700 \mathrm{~nm}$ is rejected.

Sensor's features are summarized on Table I. Among them, its good temporal resolution (below than 5ms with 1klux chip luminance), and the high dynamic range operation should be highlighted. These two features make the sensor highly competitive processing and monitoring flame activity. It should be also remarked its low output data flow, operating as a NIR imager. Only pixels exposed to NIR radiation will send data off-chip. In Section III, the sensor capabilities to monitor, study and detect flames will be discussed.

\section{EXPERIMENTAL RESULTS}

\section{A. Detection of flame emission levels}

Flames emit radiation within the NIR and the infrared band [3], [17]. With our NIR sensor, radiation sensing is restricted 


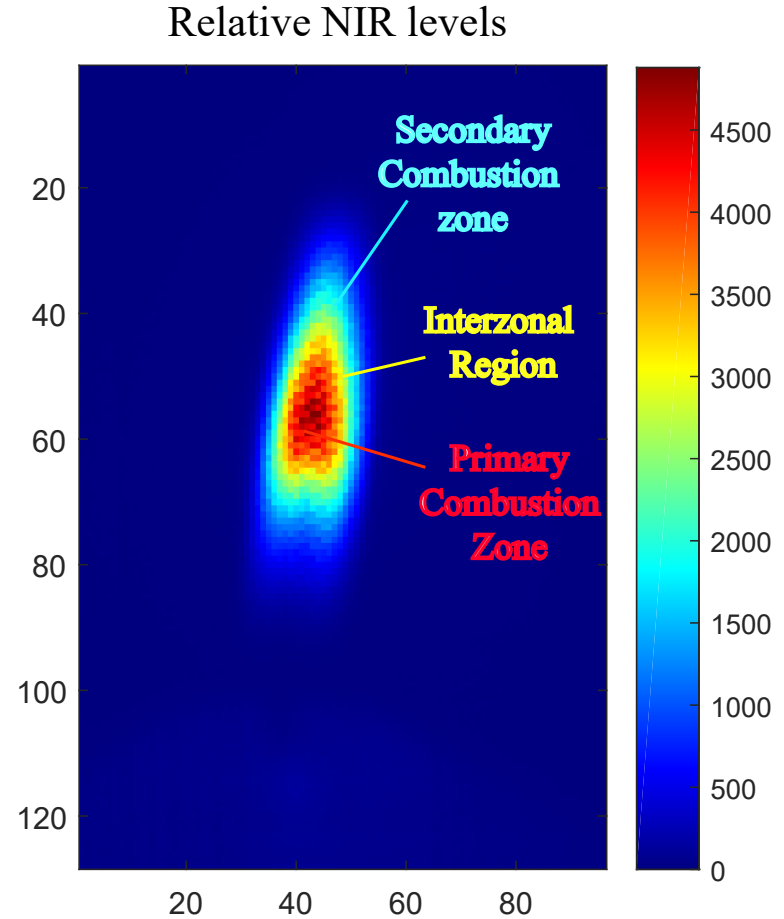

Fig. 4. Snapshot of a flame taken with the NIR sensor. Relative NIR radiation levels are indicated. Main regions inside the flame have been identified.

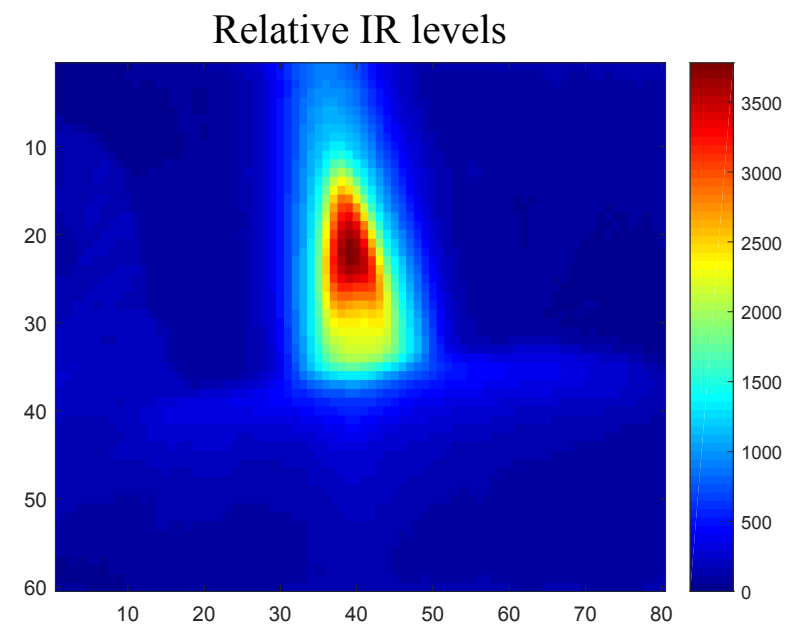

Fig. 5. Snapshot of a flame taken with the LWIR camera from Flir Lepton manufacturer. Relative IR radiation levels are indicated

TABLE II

FLir LEPTON CAMERA FEATURES

\begin{tabular}{|c|c|}
\hline Technology & Uncooled microbolometer \\
\hline Pixel Size & $17 \mu \mathrm{m}$ \\
\hline Number of pixels & $80 \times 60$ \\
\hline Power Consumption & $150 \mathrm{~mW} @$ room temperature \\
\hline Thermal sensitivity & $<50 \mathrm{mK}(0.050 \mathrm{C})$ \\
\hline Effective Frame Rate & $8.6 \mathrm{~Hz}$ \\
\hline Spectral Operation Range & $6-15 \mu \mathrm{m}$ \\
\hline
\end{tabular}

$\Delta \mathrm{T}=100 \mathrm{~ms}, 90642$ events

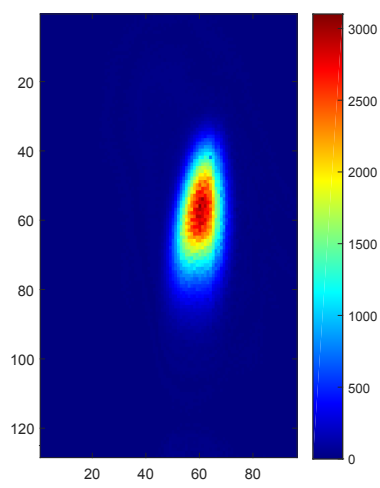

$\Delta \mathrm{T}=5 \mathrm{~ms}, 5763$ events

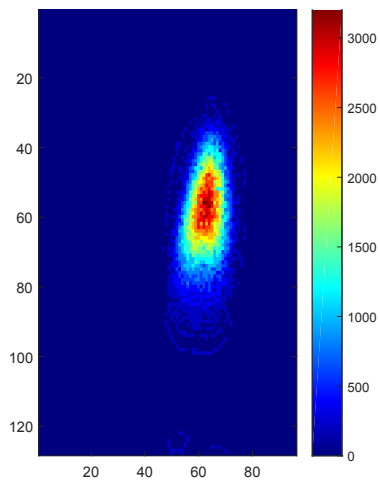

$\Delta \mathrm{T}=10 \mathrm{~ms}, 11409$ events

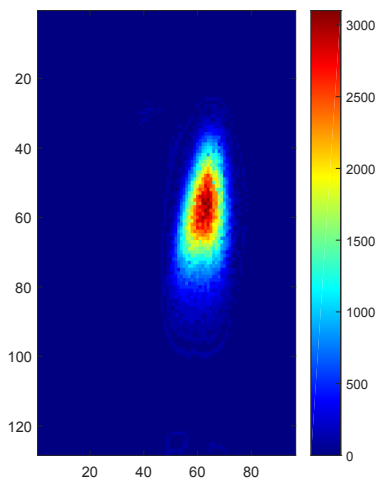

$\Delta \mathrm{T}=1 \mathrm{~ms}, 1178$ events

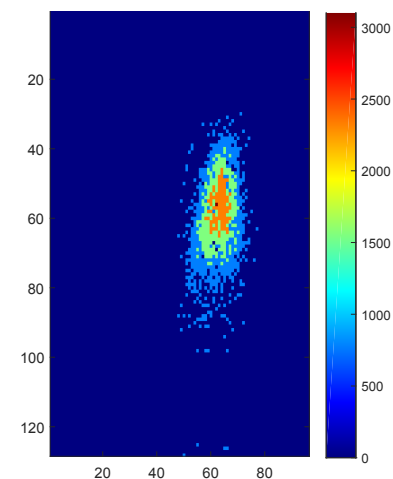

Fig. 6. Snapshots of a flame taken by the NIR AER sensor. Images are rendered after accumulating different numbers of events. For each one, the amount of time to receive the events and the number of them are indicated. Flames emission levels can be rendered much faster than employing a conventional IR camera.

to the NIR band, within the wavelength window between $[700,1100] \mathrm{nm}$. Infrared detectors detect radiation beyond the NIR band. We can wonder how the performance can be degraded filtering radiation above the NIR band, when measuring flame radiation emission levels with a CMOS HDR sensor. In this part of our investigation, we focused on determining how differ the response of the asynchronous NIR imager from the response of an IR camera, when detecting the radiation emitted by flames.

For the research, we compared the outputs of our camera with the commercial Long Wave Infrared Camera (LWIR) Flir from Lepton manufacturer. Its main features are summarized on Table II. Its spectral operation range ranges within the interval $[6,15] \mu \mathrm{m}$. For a fair comparison, we selected this device because its pixel pitch and its array resolution are alike to the implemented NIR asynchronous sensor.

We took snapshots of a gas lighter's flames. The two devices were at the same distance to the flames and mounted similar optics. Samples with the NIR sensor are displayed on Fig. 4. The main combustion regions inside the flame are indicated in the plot. Fig. 5 shows a snapshot of the same kind of flame taken with the commercial infrared camera. In this case, it is also possible to identify the main emission regions inside the flame and the hot smoke emitted by it (cyan halo above the 
flame).

Regarding the intra-scene dynamic range, we compared the relative radiation levels measured by both sensors. Intra-scene dynamic range was computed with this expression:

$$
D R(d B)=20 \cdot \log _{10}\left(I_{\max } / I_{\min }\right)
$$

Where $I_{\max }$ is the maximum and $I_{\min }$ is the minimum measured radiations level, respectively. Intra-scenes intensity levels were similar with both sensors: $71.54 \mathrm{~dB}$ with the infrared camera and $73.76 \mathrm{~dB}$ with our sensor. Therefore, both of them exhibit similar performance gauging radiation levels emitted by flames. The infrared camera can also detect radiation in the IR band emitted by the hot fumes and the hot spots around the flame, as it can be appreciated in Fig. 5. The NIR CMOS image sensor is less sensitive to temperature variations because, within the NIR band, only the radiation emitted by matter with temperature above $350^{\circ}$ can be detected [5]. For this reason, the hot regions above the flame are identified with more precision by the infrared camera.

To take a snapshot of a flame is challenging, because they flicker with frequency components up to $100 \mathrm{~Hz}$ [1]. Also wind creates flame turbulences in the scene. Thus, fast operation is desirable to avoid flame blurring, i.e. taking one snapshot containing the same flame in different regions of the visual scene. Regarding the operation speed, the NIR asynchronous sensor outperforms the results obtained with the infrared camera. The Flir Lepton device has an effective frame rate of 8.6 frames per second. It reacts mainly to temperature changes, that are much slower than irradiance variations within the visual scene.

The NIR sensor does not require the selection of an integration time. Their pixels pulse continuously with a frequency proportional to illumination. Hence, the user can decide how many spikes needs to render one image. The lower the number of spikes received the lower the amount of time to render one image. In Fig. 6, we illustrate the amount of time and the number of events required to take a snapshot of one flame. Initially, the sensor was reset. Then we released the reset signal and we recorded the incoming events. In the figure, the reconstructed image is shown, after receiving different numbers of events. It can be noticed how the location of the highest illuminated pixel changes along time due to the flame fast movement. Employing less than one millisecond, it is possible to obtain a representation of the flame radiation emitted in the NIR band with enough precision to study the flame and its turbulences.

Summarizing, studying the radiation levels emitted by flames, both sensors allows to identify the radiations levels within flames with similar precision. The IR sensor exhibits more accuracy by detecting temperature levels, especially in the boundaries of the flame, at the expense of lower operation speed and high data throughout. The IR camera can not track flame flickering with enough temporal resolution.

\section{B. Spectral Analysis of Flames}

Flames flicker with frequency components than range between DC and $100 \mathrm{~Hz}$ [1]. Their values are useful to study the
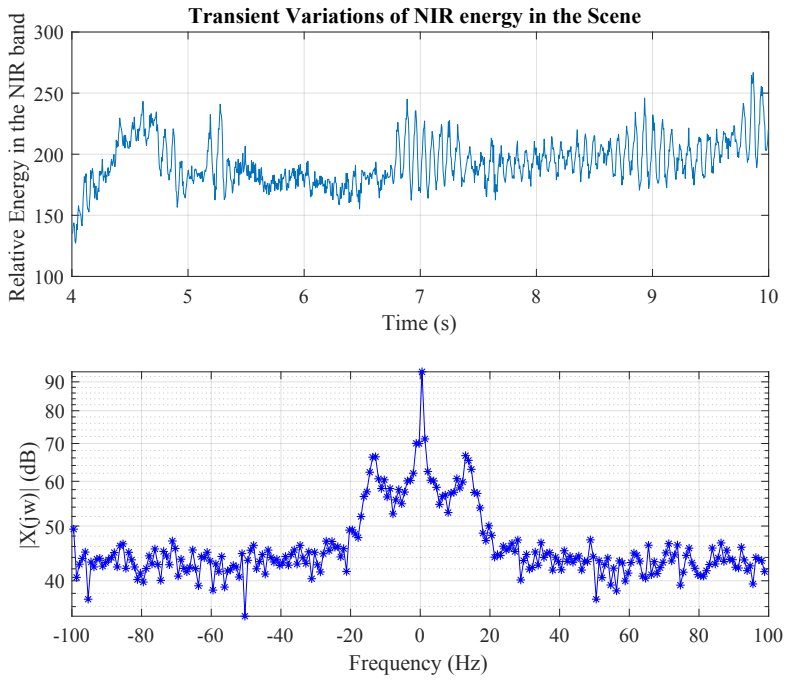

Fig. 7. Top: Transient energy variations in the NIR band provoked by a flame. (b) Fourier transform of the previous sequence. Spectral components depend on the chemical composition of the flame.

flames chemical composition and for failure detection of the flame in furnaces [1], [2]. Flickering provokes fast transient variations of the energy emitted in the NIR band. Asynchronous luminance sensors have enough temporal resolution to implement a real-time processing of flames oscillations. To do so, the energy in the NIR band of the whole scene is computed at regular time intervals of duration $T_{\text {sample }}$ :

$$
E[k]=\frac{\# \text { events } \text { Tsample }_{\text {sample }}}{T_{\text {samp }}}
$$

Where $k$ is the $k$-th time interval with duration $T_{\text {sample }}$.

Time intervals duration must satisfy that $1 / T_{\text {sample }}=$ $f_{\max } / 2$, where $f_{\max }$ is the maximum frequency component that can be detected. On top of Fig. 7, transient variations of NIR levels provoked by a flame in the visual scene are shown. On the bottom, the Fast Fourier Transform (FFT) of the previous signal was computed. Frequency main components spam between DC and $20 \mathrm{~Hz}$. Results are similar to the reported by other authors with high speed frame-based cameras [1]. The DC level depends on the flame proximity to the sensor and the flame intensity. The FFT can be updated periodically at regular time intervals that can last a few seconds. In the example, it was computed every six seconds and $T_{\text {sample }}$ was set to $5 \mathrm{~ms}$ to detect frequency components up to $100 \mathrm{~Hz}$. With this approach, we achieve a real-time computation of the flame spectrum that is not possible with convectional procedures [1] with CMOS or IR cameras. The computation load is reduced significantly because only pixels exposed to NIR radiation can generate output data continuously. With frame-based sensors, all the entire pixel matrix has to be readout are regular time intervals. Hence, the temporal resolution and the power consumption associated to data processing are degraded with respect to the proposed sensor. 


\section{Flames Detection Algorithm}

The detection of flames in the environment is not an easy task that has to be robust to false alarms [17]. We implemented a real-time algorithm to detect the presence of flames, exploiting the good temporal resolution of our asynchronous sensor. The algorithm was based on the analysis of the experimental data shown on top of Fig. 7. Flames provoke fast transient variations of the NIR levels within the visual scene with certain frequency components. These transient variations can be distinguish form other factors that provoke transient changes of the NIR levels, taking into account these two facts:

- In the majority of situations, flames flickering creates faster variations of NIR levels than objects that are moving around and reflect NIR emitted by other sources, i.g. the sun.

- Flames provoke variations of the NIR levels around their medium value with certain temporal periodicity that makes possible to distinguish them from other light sources emitting punctually in the NIR band, i.g. fast moving objects or transient perturbations in the NIR band.

The algorithm steps are as follows. First, we count the number of events, \#events $s_{T_{\text {sample }}}$, received during a time interval, $T_{\text {sample }}$. Afterwards, according Equation 3, the level of energy in the NIR band, $E[k]$ is computed. We are interested in the energy variations between adjacent samples (time intervals). By subtracting their values, $D[k]=E[k]-E[k-1]$, it is possible to obtain such variations. Also, the average illumination value in the scene will be removed by performing this operation. That is important, because the algorithm's operation should not depend on global scene illumination changes during the day.

Once we obtain the energy variations $D[k]=$ $E[k]-E[k-1]$, we study the variations of the recorded $D[k]$ values during a larger time interval $\left(T_{\text {scan }}=125-250 \mathrm{~ms}\right)$. We compute the signal envelope, maximum and the minimum values of $D[k]$ during $T_{\text {scan }}$. The difference between the maximum and the minimum values (envelope) during this interval will be the output of the fire detection algorithm, $F[n]=D_{\max }[n]-D_{\min }[n]$. If $F[n]$ is higher than a threshold value $F[n]>T H$, we will conclude that there are flames in the environment. In Section III-C.2, the value selected for the parameter will be justified. The algorithm's execution steps are listed below:

1) Every time interval, $T_{\text {sample }}$, repeat the following operations:

a) The number of events \#events $s_{T_{\text {sample }}}$ received during a time interval $T_{\text {sample }}$ are counted. Then, the global level of radiation within the NIR band, $E[k]$, is computed, according Equation 3, and stored on a buffer. b) The energy variation between consecutive samples, $D[k]$, is calculated and stored on a buffer:

$$
D[k]=E[k]-E[k-1]
$$

2) Every time interval, $T_{\text {scan }}>T_{\text {sample }}$, perform the following operations:

a) Search the maximum and the minimum values of $D[k]$ during $T_{\text {scan }}$ :

$$
\begin{gathered}
D_{\max }[n]=\max (D[k-i]), \\
D_{\min }[n]=\min (D[k-i]), \\
\text { with } \quad i=0 \ldots N, \quad N=\left\lfloor\frac{T_{\text {scan }}}{T_{\text {sample }}}\right\rfloor
\end{gathered}
$$

b) The distance between $D_{\max }[n]$ and $D_{\min }[n]$, $F[n]$ is computed

$$
F[n]=D_{\max }[n]-D_{\min }[n]
$$

c) If $F[n]$ is greater than a threshold, $T H$, a signal that represents the possible presence of fire, $A[n]$, is activated during $T_{\text {scan }}$ :

$$
A[n]= \begin{cases}1, & \text { if } F[n]>T H \\ 0, & \text { if } F[n]<T H\end{cases}
$$

3) If $A[n]$ is more time active than inactive during more than two seconds, we indicate the possible presence of flames in the visual scene.

The first loop takes into account that flames flickering creates fast fluctuations if NIR radiation levels. Objects moving around the visual scene usually cause slower variations of the NIR levels because their speed is lower than the flames flickering. Therefore, the difference $D[k]$ between consecutive values of $E[k]$ with a temporal resolution of milliseconds will be usually close to zero. It has to be noticed, that the algorithm is robust to variations of the global average illumination value, that changes much slower. Every time that $D[k]$ is computed, the average scene illumination level is removed. The method just takes into account the NIR variations around this average value.

The second loop calculates the envelope of $D[k]$ periodically. If the distance between $D_{\max }[n]$ and $D_{\min }[n]$ is higher than a certain threshold, we can infer that during such time interval there were NIR variations that could be provoked by flames.

Finally, is the signal $A[n]$ is high during more than two seconds, we emit and alarm, indicating the presence of flames. This step takes into account that flame activity has certain periodicity that is repeated along time. Thus, we can differentiate the presence of flames from temporal noise or punctual transient variations of NIR levels.

The first time constant was set to $T_{\text {sampe }}=1 \mathrm{~ms}$ to detect frequency components higher than $100 \mathrm{~Hz}$ due to flame activity. The majority of transient variations, that are usually slower, are filtered by setting this value. The second time constant, $T_{\text {scan }}$, must be set larger than the previous one. Typically, at least ten times higher. This time constant takes 
$\mathrm{T}=0 \mathrm{~ms}$
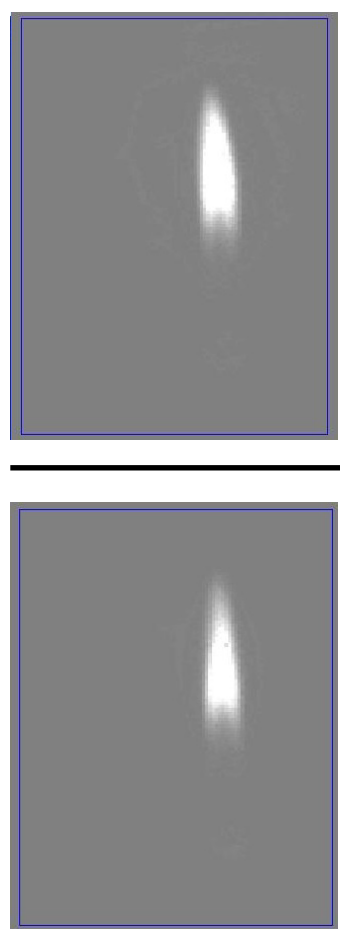

$\mathrm{T}=40 \mathrm{~ms}$
$\mathrm{T}=20 \mathrm{~ms}$

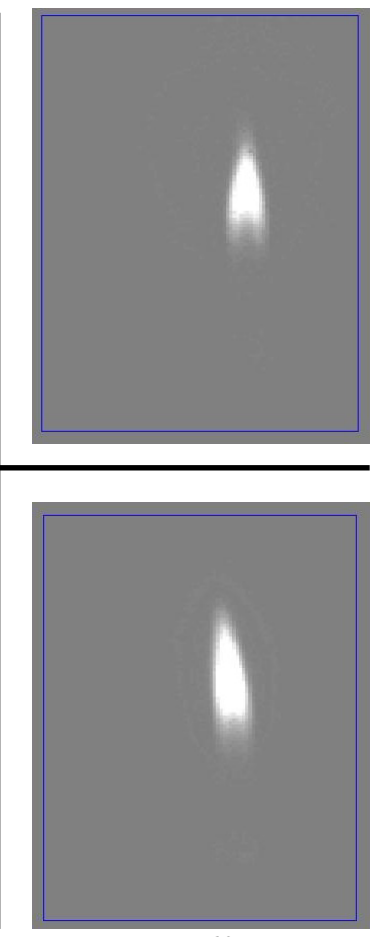

$\mathrm{T}=60 \mathrm{~ms}$
Fig. 8. jAER interface [18] showing the NIR sensor's outputs at different time stamps of 20ms. Algorithm's processing steps were implemented in Java programming language. In the snapshot, average NIR radiation levels detected by each pixel are shown. It can be noticed two effects: flame flickering and fast flame movement due to turbulences.

into account the periodicity and the repeatability of the NIR variations due to flames. Punctual transient variations are excluded, by analyzing the algorithm outputs during several periods of duration equal to $T_{\text {scan }}$.

1) Algorithm's performance: The devised flame detection algorithm computational load is compatible with real-time image processing. We used the programmable jAER interface [18] to display the sensor's outputs and to run the algorithm's processing steps. In Fig. 9, there is a flame snapshot, taken with the sensor. It can be appreciated a flame and the hot regions above it, emitting NIR radiation. This interface gives flexibility to debug and test the flame detection algorithms, before migrating them to a microcontroller or an FPGA. It is also possible to export data recordings.

To illustrate the algorithm's performance, we run it in three different situations: with and without flames, and in a visual scene with people and objects moving around. The algorithm's parameters were set as follows: $T_{\text {sample }}=5 \mathrm{~ms}$, $T_{\text {scan }}=250 \mathrm{~ms}$, and $T H=1,000$. An alarm indicating the presence of flames was triggered whenever the variable $F[n]>$ $T H$ was more time active than inactive during two seconds.

Fig. 9 the algorithm's outputs with the recording with flames shown in Fig. 8. Less than $5 \%$ pixels in the whole array were illuminated by the flame. On top of the Figure 9, the measured levels of NIR radiations, $E[k]$, are shown. These values were computed at regular time intervals of duration $T_{\text {sample }}=5 \mathrm{~ms}$.
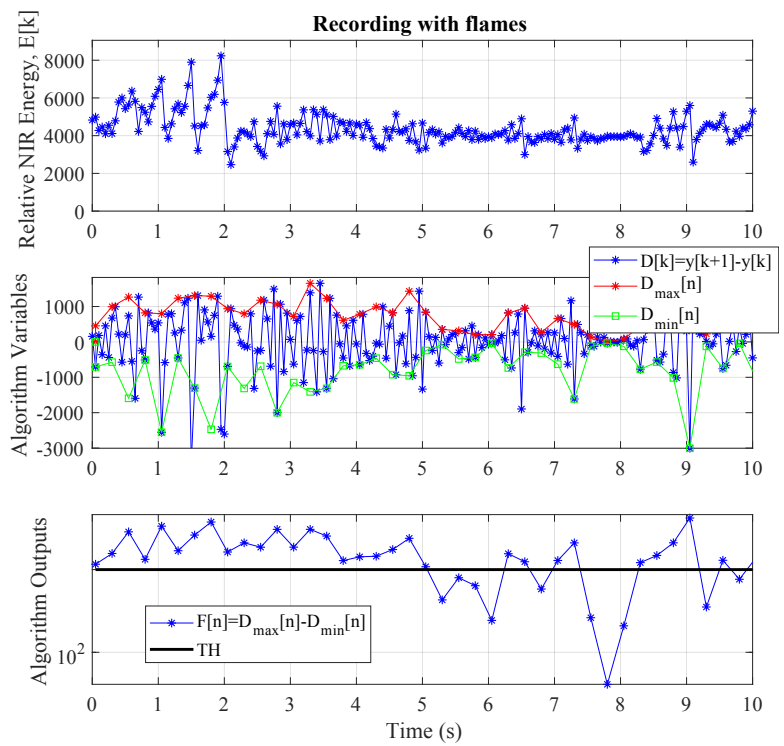

Fig. 9. Measured transient NIR levels and flame detection algorithm's outputs. Data was recorded in a visual scene with flames.
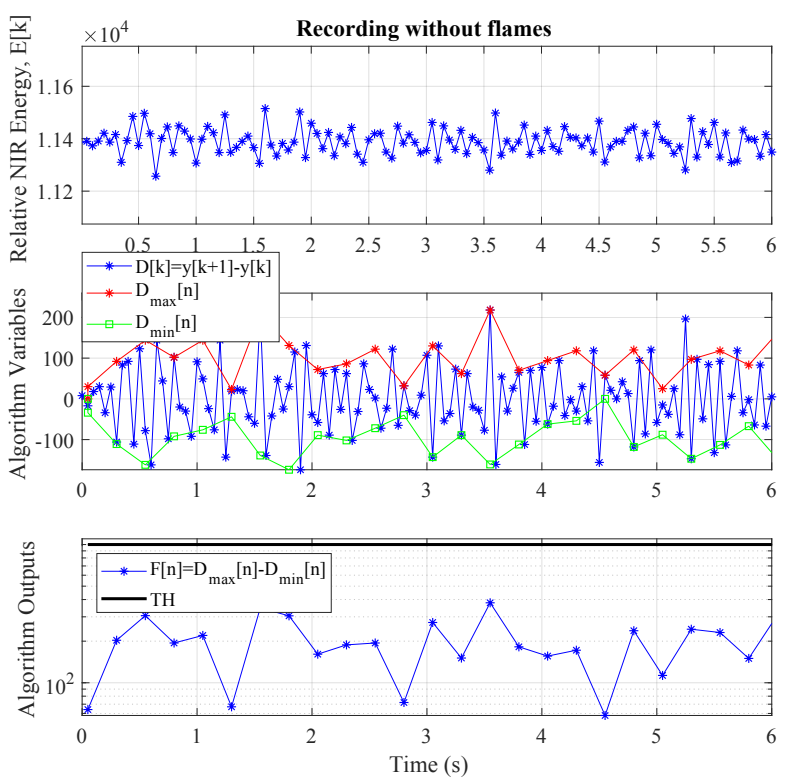

Fig. 10. Measured transient NIR levels and flame detection algorithm's outputs. Data was recorded in a visual scene without flames.

In the middle plot, the difference between consecutive estimations of the NIR energy, $D[k]=E[k]-E[k-1]$, is shown. We plotted the values of the envelope of the signal $D_{\max }[n]$ and $D_{\min }[n]$, that were computed at regular time intervals of duration $T_{\text {scan }}$. For this reason, we use different indexes for them, $k$ and $n$, respectively. On the bottom plot, we display the distance between $D_{\max }[n]$ and $D_{\min }[n]$. We generate an alarm when $F[n]$ exceeds the threshold $T H$ more than two seconds.

In Fig. 10, we appreciate the NIR levels and the algorithm's outputs, corresponding to a visual scene without flames. The algorithm's outputs are always below the limit established to 

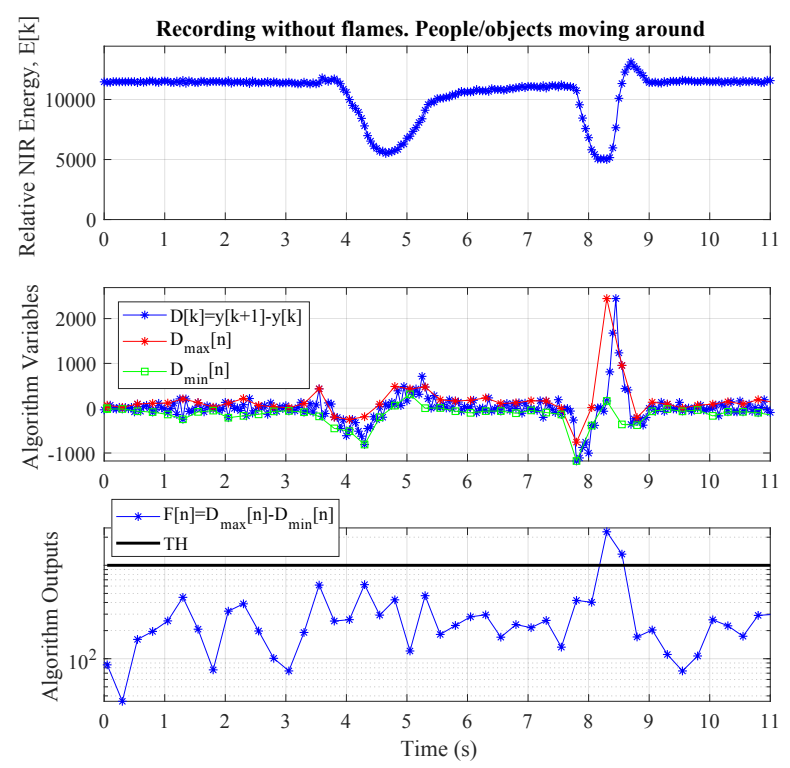

Fig. 11. Measured transient NIR levels and flame detection algorithm's outputs. Data was recorded in a visual scene with NIR sources moving around.

indicate the flame detection. NIR levels variations are due to the sensor Fixed-Pattern-Noise (FPN).

Finally, in Fig. 11, we analyze data recordings in a visual scene with people and objects moving around. The visual scene was illuminated with a LED array emitting in the NIR band to assure that the elements within the visual scene were able to emit (reflect) NIR radiation that could be detected by the sensor. After recording data during four seconds, a person walking crossed the sensor's field of view. In this case, the variations of NIR levels are slow in comparison to the time constant set by the algorithm. Hence, the algorithm discards these variations as possible presence of flames. Later on, we moved an object crossing the visual field at faster speed. In this case, it causes transient variations of the NIR levels that are detected. However, the algorithm checks the repeatability of the NIR variations. This discards, in this case, the presence of flames. Moreover, the algorithm was also tested with data sets recorded in an office with people working and moving around. No flame alarms were triggered.

2) Algorithm's limitations: We identified two main factors that limit the algorithm's efficiency. The first one is the presence of NIR light sources provoking periodic variations of NIR levels. For instance, NIR illumination transient variations emitted by bulbs. These distractors can be detected because $F[n]$ values are always high and constant. Periodic light sources can also be discarded by correlating their spectral response with the expected spectral response of a flame (see bottom plot at Fig. 7). The penalty is a higher computational load to calculate the FFT periodically.

The second limitation is referred to the sensor's FixedPattern-Noise (FPN=2.6\%, for the reported sensor). This provokes random variations of the pixels spiking frequencies around its average value. To avoid false alarms, NIR transient variations associated to flames should be higher than the caused by FPN. In outdoors environments with very bright sunlight, the average level of the NIR levels of the visual scene increases significantly. In such circumstances, NIR variations around the medium value are more difficult to differentiate because they can be similar to the fluctuations due to FPN. Therefore, FPN imposes a limit for the minimum NIR transient variations that can be detected.

The algorithm's threshold value, $T H$, is chosen trying to reach an equilibrium between the algorithm's sensitivity to NIR fluctuations and false alarms inferred by FPN. In the algorithm executions displayed in the article, the algorithm threshold value was chosen to be $T H=1,000$. This value is approximately twice the value of the algorithm output without flames, with an average illumination value of 400lux (shown on the bottom of Fig. 10). This implies that, under these circumstances, any NIR variation generated by a flame that is two times higher than the FPN variations will be detected. The intensity of the NIR variations will depend on many factors: distance from the flame to the camera, number of pixels exposed to NIR radiation, the flame intensity, etc.

\section{CONCLUSIONS}

We have demonstrated the capabilities of a NIR asynchronous image sensor measuring flame radiation, monitoring NIR transient changes, and detecting flame activity. Such tasks have been traditionally relegated to IR and NIR frame-based imagers. The experimental results provided show that spiking NIR sensors are highly competitive in terms of temporal resolution, data throughout, and dynamic range. They outperform IR and NIR frame-based CMOS imagers operation speed. Their high dynamic range allows to measure and identify flames radiation levels with an acceptable precision. Exploiting our sensor accurate temporal resolution, we monitored flames flickering and we presented a flame detection procedure. As a future work, we plan to refine the developed flame detection algorithms for asynchronous luminance sensors and implement them on an FPGA.

\section{REFERENCES}

[1] Y. Yan, C. M., and H. R., "Monitoring of oscillatory characteristics of pulverized coal flames through image processing and spectral analysis," IEEE Transactions on Instrumentation and Measurement, vol. 55, pp. 226-231, February 2006.

[2] A. R. Jones, "Flame failure detection and modern boilers," Journal of Physics E: Scientific Instruments, vol. 21, no. 10, p. 921, 1988. [Online]. Available: http://stacks.iop.org/0022-3735/21/i=10/a=001

[3] S. Briz, A. de Castro, J. Aranda, J. Melendez, and F. Lopez, "Reduction of false alarm rate in automatic forest fire infrared surveillance systems," Remote Sensing of Environment, pp. 19-29, 2003.

[4] J. Cheon, J. Lee, I. Lee, Y. Chae, Y. Yoo, and G. Han, "A single-chip CMOS smoke and temperature sensor for an intelligent fire detector," IEEE Sensors Journal, vol. 9, pp. 625-628, 2009.

[5] Y. L. Maoult, T. Sentenac, J. Orteu, and J. P. Arcens, "A new approach based on a low cost CCD camera in the near infrared," Fire Detection, pp. 193-206, 2007.

[6] P. Bendiscio, F. F. J, P. Malcovat, F. Malobert, M. Polett, and R. Valacca, "A CMOS integrated infrared radiation detector for flame monitoring," ISCAS, vol. 6, pp. 625-628, 1998.

[7] J. Fernandez-Berni, R. Carmona-Galan, J. F. Martinez-Carmona, and A. Rodriguez-Vazquez, "Early forest fire detection by vision-enabled wireless sensor networks," Int. Journal of Wildland Fire, July 2012.

[8] Q. Li, Q. Hao, and K. Zhang, "Smart wireless video sensor network for fire alarm," 6th International Conference on Wireless Communications Networking and Mobile Computing (WiCOM), pp. 1-4, September 2010. 
[9] A. Darmont, "Methods to extend the dynamic range of snapshot active pixel sensors," in Proceedings of SPIE Vol. 6816, 681603 (2008), February 2008.

[10] J. Leñero-Bardallo, D. Bryn, and P. Häfliger, "Flame monitoring with an AER color vision sensor," in Circuits and Systems (ISCAS), 2013 IEEE International Symposium on, May 2013, pp. 2404-2407.

[11] E. Culurciello, R. Etienne-Cummings, and K. Boahen, "A biomorphic digital image sensor," Solid-State Circuits, IEEE Journal of, vol. 38, no. 2, pp. 281-294, Feb 2003.

[12] J. A. Leñero-Bardallo, R. Carmona-Galán, and A. Rodríguez-Vázquez., "A bio-inspired vision sensor with dual operation and readout modes," Sensors Journal, IEEE, vol. 16, no. 2, pp. 1-14, January 2016.

[13] J. A. Leñero-Bardallo, D. Bryn, and P. Häfliger, "Bio-inspired asynchronous pixel event tricolor vision sensor," Biomedical Circuits and Systems, IEEE Transactions on, vol. 8, no. 3, pp. 345-357, June 2014.

[14] J. A. Leñero-Bardallo, R. Carmona-Galán, and A. Rodríguez-Vázquez, "A wide linear dynamic range image sensor based on asynchronous self-reset and tagging of saturation events," IEEE Journal of Solid-State Circuits, vol. 52, no. 6, pp. 1605-1617, April 2017.

[15] M. Silvilotti, "Wiring considerations in analog VLSI systems with application to field-programmable networks," Ph.D. dissertation, Cal. Inst. of Tech., Pasadena, California, 1991.

[16] P. Häfliger, "A spike based learning rule and its implementation in analog hardware," Ph.D. dissertation, ETH Zürich, Switzerland, 2000, http://www.ifi.uio.no/ hafliger.

[17] B. C. Arrue, A. Ollero, and J. R. M. de Dios, "An intelligent system for false alarm reduction in infrared forest-fire detection," IEEE Intelligent Systems and their Applications, vol. 15, no. 3, pp. 64-73, May 2000.

[18] “jAER open source project," http://sourceforge.net/projects/jaer/. 